\title{
Assessment of Lipid Quality and Composition of Commercial Infant Milk Formulas in Mexico: Emphasis on Trans Fatty Acid Isomers
}

\author{
Ayala-Moreno María del Rosario', \\ Fernández-Callejas José María Salvador ${ }^{1}$, \\ Maldonado-Hernández Jorge ${ }^{2 *}$ \\ ${ }^{1}$ Food and Health Research Group, School of Chemistry, Universidad La Salle, \\ Mexico City, Mexico \\ ${ }^{2}$ Mass Spectrometry Laboratory, Research Unit in Medical Nutrition, Pediatric Hospital, National Medical Center \\ "Siglo 21", Mexican Institute of Social Security, \\ Mexico City, Mexico \\ Email: rosario.ayala@ulsa.mx, josemasafer@gmail.com, "jormh@yahoo.com.mx
}

Received 23 December 2015; accepted 24 April 2016; published 27 April 2016

Copyright (C) 2016 by authors and Scientific Research Publishing Inc.

This work is licensed under the Creative Commons Attribution International License (CC BY). http://creativecommons.org/licenses/by/4.0/

cC) (i) Open Access

\begin{abstract}
Objective: To assess the lipid quality focusing on trans fatty acids (TFA) content of standardized milk formulas marketed in Mexico for infants aged from 0 to 36 months. Material and Methods: A total of 27 infant formulas from eight different leading brands were analyzed. Nine of them belonged to stage 1 (age < 6 months), nine to stage 2 (age 6 to 12 months) and eight to stage 3 (age > 12 months). Acquired products were treated by duplicate for extraction of total lipid content with the modified Folch method before their expiration date. Fatty acids were esterified in an alkaline medium followed by an acid-catalyzed esterification. Analysis was performed on a gas chromatograph (5890 Series II; Hewlett-Packard, USA) with a flame ionization detector. Results: Thirty-four fatty acids (C8 to C22) were identified. Most products complied with ESPHAGAN compositional requirements. Only one product exceeded the suggested limit $(>3 \%)$ for TFA. Long chain polyunsaturated fatty acids (LC-PUFAs) content was consistently meager $(\approx 78 \%)$, with low amounts of arachidonic $(<0.35 \%)$ and docosahexaenoic acids $(<0.2 \%)$. Conclusion: Most milk formulas complied with ESPHAGAN global recommendations. The content of TFA and LC-PUFAs was scarce in the majority of samples.
\end{abstract}

\footnotetext{
${ }^{*}$ Corresponding author.
}

How to cite this paper: del Rosario, A.-M.M., Salvador, F.-C.J.M. and Jorge, M.-H. (2016) Assessment of Lipid Quality and Composition of Commercial Infant Milk Formulas in Mexico: Emphasis on Trans Fatty Acid Isomers. Food and Nutrition Sciences, 7, 273-283. http://dx.doi.org/10.4236/fns.2016.74029 


\section{Keywords}

\section{Infant Formulas, Milk, Lipid Quality, Trans Fatty Acids}

\section{Introduction}

Breastfeeding confers unique biological, psycho-affective and socio-economic benefits to the infant and the mother. The American Academy of Pediatrics recommends exclusive breastfeeding (EBF) during the first six months of life and sustained until the first year of age with the gradual inclusion of complementary foods [1]. Global prevalence of EBF in developing countries increased from 33\% to 39\% between 1995 and 2010 [2]. Despite the numerous benefits of this practice, the observed increment is still quite modest. In the case of Mexico, the prevalence of EBF decreased dramatically from 22.3\% to 14.5\% between 2006 and 2012 [3]. Interestingly, during the same period of time, an increment in the consumption of infant formulas or other milk-like products (4\%) and water (4\%), was observed in infants under 6 months of age [3].

On 2004, the Codex Committee on Nutrition and Food for Specially Dietary Uses, asked the European Society for Pediatric Gastroenterology, Hepatology and Nutrition (ESPGHAN) to propose compositional requirements for in infant formulas to attain normal growth and development. Regarding lipids, total fat content must be between 4.4 to $6 \mathrm{~g} / 100 \mathrm{kcal}$, equivalent to $40 \%$ - 54\% of the total energy requirement. Linoleic acid (C18:2n-6) and $\alpha$-linolenic acid (C18:3n-3) must provide at least $2.7 \%$ and $0.45 \%$ of the total energy intake $(0.3 \mathrm{~g} / 100 \mathrm{kcal}$ and $0.05 \mathrm{~g} / 100 \mathrm{kcal}$, respectively). Linoleic acid $/ \alpha$-linolenic acid ratio is acceptable in the range of 5 - 15 to 1 . The sum of saturated fatty acids Lauric (C12:0) and Myristic (C14:0) should not exceed 20\% of the total fatty acids [4].

Long chain polyunsaturated fatty acids (LC PUFA) have a chain length of 20 or more carbon atoms, nutritionally the most relevant ones are omega- 3 and omega- 6 fatty acids. Omega-3 LC PUFA encompass eicosapentaenoic and docosahexaenoic acids (EPA: C20:5n-3 and DHA: C22:6n-3, respectively); arachidonic acid (AA: C20:4n-6) is the main Omega-6 LC PUFA. The addition of DHA and AA to the infant formulas may support visual and cognitive development [5]-[7]. Several authors recommend the addition of DHA to achieve a concentration above $0.2 \%$ but below $0.5 \%$ of the total fatty acids, the content of AA should exceed $0.35 \%$ with a maximum of $1 \%$ [8].

Trans Fatty Acids (TFA) are unsaturated fatty acids with at least one double bond in trans configuration. TFA are mostly produced during industrial processes of deodorization, refinement and partial hydrogenation of vegetable oils. The most abundant TFA generated during these industrial processes are elaidic (C18:1n-9t) and linoelaidic acids (C18:2n-6t) [9]. TFA can also be formed naturally throughout the rumination process, so small amounts of them are present in milk, dairy products and meat. Vaccenic acid (C18:1n-7t) from which conjugated linoleic acid is formed (C18:2n-7t), is the predominant trans-isomer in ruminants [10]. Recent studies in adults and in pediatric populations have shown that a high dietary intake of industrial origin TFA increases serum low-density lipoprotein cholesterol (LDL-C) and TG, and decreases serum HDL-C [11] [12]; besides, the human lipase enzyme is specific for cis configuration and is ineffective with trans configuration, so trans fat remains in the bloodstream for a much longer period of time. In contrast, some authors report that natural origin TFA (C18:1n-7t and C18:2n-7t) confer protective effects against metabolic disorders [13]. The acceptable limit for total TFAs is $3 \%$ of the total fat content.

In Mexico and other developing countries, infant formulas represent a staple nourishment to cover nutritional requirements [14] [15]. Because lipids have a transcendental role in growth and other important biological events such as neurological, immune and cardiovascular development, consequently the assessment of lipid quality is important to verify the adequacy to global recommendations. Therefore, the aim of this study was to determine the lipid quality of standard milk formulas marketed in Mexico for infants from 0 months to 3 years of age emphasizing in TFA content. Lipid quality was assessed according to ESPHAGAN global recommendations.

\section{Methods}

\subsection{Sample Selection}

In order to identify available milk infant formulas research was made on internet databases and through ground- 
work visiting supermarkets and pharmacies in Mexico City’s Metropolitan area during December 2011. After identifying commercial brands and price ranges, products containing milk as the main protein source were selected. Specialized infant formulas such as soy-based and lactose-free products were excluded from the study. Sample selection included formulas from stage 1 also known as "start formulas", which are recommended from the first day of birth to 6 months of age, stage 2 formulas or "continuous formulas" which are recommended for feeding infants from 6 months to 1 year of age, and stage 3 or "growth formulas" recommended for children over one year of age, according to the consensus of experts on nutritional aspects of infant formulas [16]. Products were purchased and their fat content, lot number, expiration date, country of origin, ingredients and instructions for preparation were registered as reported on labels.

\subsection{Lipid Extraction}

Acquired products were treated by duplicate for extraction of total lipid content with the modified Folch method before their expiration date [17]. Powdered formulas were weighed with an analytical balance and reconstituted according to the instructions provided by the manufacturer. Reconstituted samples were homogenized and an aliquot was drawn into a test tube for fat extraction. Afterward, lipid content was determined by weighting the dried residue in the vials. Samples were stored at $-70^{\circ} \mathrm{C}$ until the next step of analysis.

\subsection{Esterification of Fatty Acids}

Several protocols for the preparation of fatty acid methyl esters (FAME) were reviewed; a combined method described by Kramer et al. was selected because it has the highest efficiency for transfatty acid extraction, this technique consists of two consecutive reactions: a transesterification in an alkaline medium followed by an acidcatalyzed esterification [18]-[20].

Lipids $(20 \mathrm{mg}$ ) were added with $0.5 \mathrm{~mL}$ of dry toluene chromatographic grade and $1 \mathrm{~mL}$ of $0.5 \mathrm{~N}$ methanolic sodium methoxide (Sigma-Aldrich Chemie GmbH, Germany). Samples were shaken for 30 seconds and placed in a $55^{\circ} \mathrm{C}$ heating block (Reacti-Therm II, Pierce Biotechnology, USA) during 15 minutes. Once the reaction was finished, samples were removed from heat and cooled down with tap water. Then, $1 \mathrm{~mL}$ of a methanolic solution containing $14 \%$ boron trifluoride (Sigma-Aldrich Chemie GmbH, Germany) and $250 \mu \mathrm{L}$ of internal standard solution (100 mg/mL margaric acid in methanol, Sigma-Aldrich Chemie GmbH, Germany) were added. Samples were shaken again for 30 seconds and heated under the same conditions. After cooling down with tap water, $1 \mathrm{~mL}$ of isooctane (J. T. Baker, USA) and $4 \mathrm{~mL}$ of an aqueous saturated solution of sodium chloride were added to the samples. After shaking for 2 minutes vials were centrifuged at $628.875 \mathrm{~g}$ and at $4^{\circ} \mathrm{C}$ for 10 minutes. The upper organic layer was recovered and solvent was then removed by a stream of nitrogen (ultra-high purity). Dried FAME were weighed and stored at $-70^{\circ} \mathrm{C}$ until gas-chromatographic analysis was performed.

\subsection{Analysis of FAME by Gas Chromatography}

Groups of samples were thawed and allowed to reach room temperature, then total FAME were dissolved in 1 $\mathrm{mL}$ of isooctane (chromatographic grade), shaking vigorously to ensure homogeneity. An aliquot was added to empty vials containing an appropriate volume of isooctane to reach a concentration of $1 \mathrm{mg} / \mathrm{mL}$. The diluted samples were gently shaken and injected into the gas chromatograph. Room temperature was controlled at $20^{\circ} \mathrm{C}$ during each injection sequence. Infant formulas were analyzed by duplicate in groups according to the stage (1, 2 or 3) with a blank and a control sample. Analysis was performed on a gas chromatograph (5890 Series II; Hewlett-Packard, USA) with a flame ionization detector (FID). Fatty acids were separated using HP-88 capillary column (100 m × 0.25 mm ID; Agilent Technologies, Inc., USA). Carrier gas was ultra-high purity $\mathrm{He}_{2}$, with a linear velocity of $1 \mathrm{ml} / \mathrm{min}$. The following temperature program was established after several attempts in order to improve resolution of peaks; initial: $70^{\circ} \mathrm{C}$ with a $8 \mathrm{~min}$ hold; ramp: $30^{\circ} \mathrm{C} / \mathrm{min}$ to $175^{\circ} \mathrm{C}$ with a $1 \mathrm{~min}$ hold, $1.2^{\circ} \mathrm{C} / \mathrm{min}$ to $230^{\circ} \mathrm{C}$ with a $5 \mathrm{~min}$ hold.

\subsection{Quality Control}

One blank and one control sample were included in each methylation lot. Blank samples were given the same treatment as lipid extracts. Control samples contained an aliquot of vegetal oil from a refrigerated stock supply and were processed as mentioned above. Linoleic/Linolenic acid ratio in vegetal oil was used to determine the 
coefficient of variation among different batches.

\subsection{Statistical Analysis}

Data are expressed as the mean value of each fatty acid (g) analyzed by duplicate, per $100 \mathrm{~g}$ of fat. Linoleic (LA) and Linolenic (ALA) acids data were transformed to g per $100 \mathrm{kcal}$ of the reconstituted formula. ESPHAGAN fat compositional requirements of the analyzed formulas (Table 5) are reported as \% w/w. Finally, fat compositional comparisons between different stages were performed with ANOVA.

\section{Results}

We analyzed a total of 27 infant formulas of eight different leading brands. Nine of them belonged to stage 1 ( $<6$ months), nine to stage 2 (6 to 12 months) and eight to stage 3 (>12 months). Table 1 lists the commercial name, brief description, manufacturer, and country of origin of the analyzed products. Thirty-four fatty acids ranging from C8 to C22 chain lengths were identified. The detailed fatty acid profiles of each formula are presented according to the nutrition stage they correspond to in Table 2 (stage 1) Table 3 (stage 2) and Table 4 (stage 3).

Table 1. Selected and analyzed infant formulas.

\begin{tabular}{|c|c|c|c|}
\hline Product name & Description & Manufacturer & Country of origin \\
\hline \multicolumn{4}{|c|}{ Stage 1} \\
\hline Enfamil premium 1 & Infant formula from birth to 6 months & Mead Johnson & Mexico \\
\hline Equate 1 & Infant formula from birth to 6 months & PBM Nutritionals & United States \\
\hline Frisolac 1 & Infant formula from birth to 6 months & PiSA & Netherlands \\
\hline Good start supreme 1 & Infant formula from birth to 6 months & Nestlé & Mexico \\
\hline Nan 1 & Infant formula from birth to 6 months & Nestlé & Mexico \\
\hline Novamil 1 & Infant formula from birth to 6 months & Bayer & France \\
\hline Nutra enfant 1 & Infant formula from birth to 6 months & PBM Nutritionals & United States \\
\hline Similac 1 & Infant formula from birth to 6 months & Abbott & Ireland \\
\hline SMA gold 1 & Infant formula from birth to 6 months & Wyeth & Mexico \\
\hline \multicolumn{4}{|c|}{ Stage 2} \\
\hline Enfapro premium 2 & Infant formula for older babies $6+$ months & Mead Johnson & Mexico \\
\hline Equate 2 & Infant formula for babies from 6 to 12 months & PBM Nutritionals & United States \\
\hline Friso 2 & Infant formula for babies from 6 to 12 months & PiSA & Netherlands \\
\hline Good start supreme 2 & Infant formula for babies from 6 to 12 months & Nestlé & Mexico \\
\hline Nan 2 & Infant formula for older babies $6+$ months & Nestlé & Mexico \\
\hline Novamil 2 & Infant formula for babies from 6 to 12 months & Bayer & France \\
\hline Nutra enfant 2 & Infant formula for older babies $6+$ months & PBM Nutritionals & United States \\
\hline Promil gold 2 & Infant formula for babies from 6 to 12 months & Wyeth & Mexico \\
\hline Similac gain 2 & Infant formula for babies from 6 to 12 months & Abbott & Denmark \\
\hline \multicolumn{4}{|c|}{ Stage 3} \\
\hline Enfagrow premium 3 & Infant formula for toddlers & Mead Johnson & Mexico \\
\hline Equate 3 & Infant formula for toddlers & PBM Nutritionals & United States \\
\hline Friso 3 & Infant formula for toddlers from 1 to 3years & PiSA & Netherlands \\
\hline Gain plus 3 & Infant formula for toddlers from 1 to 3years & Abbott & Mexico \\
\hline Good care supreme 3 & Infant Formula for older babies $12+$ months & Nestlé & Ireland \\
\hline Nan 3 development & Infant Formula for older babies $10+$ months & Nestlé & Mexico \\
\hline Nutra enfant 3 & Infant formula for toddlers & PBM Nutritionals & United States \\
\hline Progress gold 3 & Infant formula for toddlers from 1 to 3years & Wyeth & Mexico \\
\hline
\end{tabular}


Table 2. Detailed fatty acid profile of stage 1 formulas.

\begin{tabular}{|c|c|c|c|c|c|c|c|c|c|}
\hline \multirow{2}{*}{ Chain length (trivial name) } & \multicolumn{9}{|c|}{$\mathrm{g}$ of fatty acid/100g fat } \\
\hline & EP1 & E1 & F1 & GSS1 & NN1 & N1 & NE1 & S1 & SMAG1 \\
\hline C8:0 (caylic) & 0.34 & 0.58 & 0.18 & 0.18 & 0.40 & 0.63 & 0.60 & 0.77 & 0.13 \\
\hline C10:0 (caproic) & 0.88 & 1.15 & 0.44 & 0.69 & 1.03 & 1.47 & 1.14 & 1.53 & 0.68 \\
\hline C11:0 & 0.00 & 0.00 & 0.00 & 0.00 & 0.00 & 0.00 & 0.00 & 0.00 & 0.00 \\
\hline C12:0 (lauric) & 11.58 & 9.60 & 6.13 & 10.53 & 9.65 & 12.92 & 9.60 & 13.02 & 11.09 \\
\hline C13:0 & 0.03 & 0.00 & 0.00 & 0.02 & 0.00 & 0.00 & 0.00 & 0.00 & 0.03 \\
\hline C14:0 (myristic) & 4.91 & 4.43 & 2.98 & 4.55 & 4.57 & 6.11 & 4.55 & 5.51 & 4.62 \\
\hline C15:0 & 0.07 & 0.08 & 0.08 & 0.10 & 0.09 & 0.07 & 0.08 & 0.05 & 0.07 \\
\hline C16:0 (palmitic) & 23.95 & 22.96 & 25.82 & 27.60 & 26.34 & 24.99 & 23.10 & 8.40 & 21.38 \\
\hline C18:0 (estearic) & 3.88 & 4.40 & 3.51 & 4.17 & 4.04 & 3.94 & 4.26 & 3.50 & 4.51 \\
\hline C20:0 (arachidic) & 0.37 & 0.34 & 0.43 & 0.38 & 0.41 & 0.31 & 0.33 & 0.30 & 0.39 \\
\hline C21:0 & 0.00 & 0.00 & 0.00 & 0.00 & 0.00 & 0.00 & 0.00 & 0.03 & 0.00 \\
\hline C22:0 (behenic) & 0.16 & 0.16 & 0.15 & 0.12 & 0.14 & 0.18 & 0.17 & 0.53 & 0.17 \\
\hline C23:0 & 0.00 & 0.00 & 0.00 & 0.00 & 0.00 & 0.00 & 0.00 & 0.02 & 0.00 \\
\hline C24:0 (lignoceric) & 0.03 & 0.06 & 0.07 & 0.10 & 0.06 & 0.00 & 0.07 & 0.00 & 0.00 \\
\hline Sum of Saturated & 46.19 & 43.74 & 39.78 & 48.43 & 46.74 & 50.62 & 43.90 & 33.68 & 43.06 \\
\hline C14:1 (myristoleic) & 0.03 & 0.00 & 0.00 & 0.03 & 0.00 & 0.00 & 0.00 & 0.01 & 0.00 \\
\hline C15:1 & 0.00 & 0.00 & 0.00 & 0.00 & 0.00 & 0.00 & 0.00 & 0.00 & 0.00 \\
\hline C16:1 (palmitoleic) & 0.21 & 0.19 & 0.27 & 0.25 & 0.24 & 0.15 & 0.19 & 0.14 & 0.17 \\
\hline $\mathrm{C} 17: 1$ & 0.05 & 0.05 & 0.06 & 0.06 & 0.05 & 0.05 & 0.06 & 0.05 & 0.06 \\
\hline C18:1n-9 (oleic) & 34.90 & 34.96 & 43.42 & 32.74 & 35.20 & 26.40 & 34.89 & 42.37 & 36.54 \\
\hline C20:1 & 0.28 & 0.17 & 0.44 & 0.23 & 0.33 & 0.19 & 0.18 & 0.21 & 0.34 \\
\hline C22:1n-9 (euricic) & 0.00 & 0.00 & 0.00 & 0.00 & 0.00 & 0.00 & 0.00 & 0.00 & 0.00 \\
\hline C24:1n-9 (nervonic) & 0.00 & 0.03 & 0.04 & 0.00 & 0.00 & 0.00 & 0.02 & 0.00 & 0.04 \\
\hline Sum of MUFAS (cis) & 35.49 & 35.40 & 44.23 & 33.31 & 35.82 & 26.79 & 35.33 & 42.79 & 37.15 \\
\hline C18:2n-6c (linoleic) & 15.36 & 18.56 & 13.32 & 15.60 & 14.69 & 20.42 & 18.28 & 20.82 & 17.05 \\
\hline C18:3n-6 ( $\gamma$-linolenic) & 0.06 & 0.00 & 0.00 & 0.00 & 0.00 & 0.00 & 0.00 & 0.03 & 0.02 \\
\hline $\mathrm{C} 20: 2$ & 0.03 & 0.00 & 0.00 & 0.00 & 0.00 & 0.00 & 0.00 & 0.00 & 0.00 \\
\hline C20:3n-6 (dihomo- $\gamma$-linolenic) & 0.08 & 0.00 & 0.00 & 0.04 & 0.04 & 0.00 & 0.00 & 0.05 & 0.05 \\
\hline C20:4n-6 (Arachidonic) & 0.70 & 0.19 & 0.22 & 0.37 & 0.21 & 0.00 & 0.20 & 0.44 & 0.37 \\
\hline $\mathrm{C} 22: 2$ & 0.00 & 0.00 & 0.00 & 0.00 & 0.00 & 0.00 & 0.00 & 0.00 & 0.00 \\
\hline Sum of PUFAS n-6 & 16.24 & 18.75 & 13.53 & 16.01 & 14.94 & 20.42 & 18.48 & 21.35 & 17.49 \\
\hline C18:3n3 (linolenic) & 1.49 & 1.70 & 1.90 & 1.52 & 1.95 & 1.95 & 1.85 & 1.56 & 1.84 \\
\hline C20:3n3 & 0.00 & 0.00 & 0.08 & 0.00 & 0.00 & 0.00 & 0.00 & 0.00 & 0.00 \\
\hline C20:5n3 (EPA) & 0.09 & 0.09 & 0.09 & 0.09 & 0.09 & 0.09 & 0.10 & 0.19 & 0.10 \\
\hline C22:6n3 (DHA) & 0.30 & 0.16 & 0.17 & 0.33 & 0.18 & 0.00 & 0.17 & 0.21 & 0.17 \\
\hline Sum of PUFAS n-3 & 1.88 & 1.95 & 2.24 & 1.94 & 2.21 & 2.03 & 2.12 & 1.96 & 2.11 \\
\hline C18:1n-9t (elaidic) & 0.13 & 0.09 & 0.22 & 0.14 & 0.14 & 0.07 & 0.11 & 0.10 & 0.13 \\
\hline C18:2n-6t (linolelaidic) & 0.00 & 0.00 & 0.00 & 0.00 & 0.00 & 0.00 & 0.00 & 0.00 & 0.00 \\
\hline Sum of non-natural TFA & 0.13 & 0.09 & 0.22 & 0.14 & 0.14 & 0.07 & 0.11 & 0.10 & 0.13 \\
\hline C18:1n7t (vaccenic) & 0.07 & 0.06 & 0.00 & 0.12 & 0.10 & 0.07 & 0.06 & 0.07 & 0.06 \\
\hline C18:2n7t (CLA) & 0.00 & 0.00 & 0.00 & 0.05 & 0.05 & 0.00 & 0.00 & 0.06 & 0.00 \\
\hline Sum of natural TFA & 0.07 & 0.06 & 0.00 & 0.17 & 0.15 & 0.07 & 0.06 & 0.13 & 0.06 \\
\hline
\end{tabular}

Data presented as mean. MUFAS: monounsaturated fatty acids, PUFAS: polyunsaturated fatty acids, TFA: trans fatty acids. EP1: Enfamil, E1: Equate 1, F1: Frisolac 1, GSS1: Good Start Supreme 1, NN1: Nan 1, N1: Novamil 1, NE1: Nutra Enfant 1, S1: Similac 1, SMAG1: SMA Gold 1. 
Table 3. Detailed fatty acid profile of stage 2 formulas.

\begin{tabular}{|c|c|c|c|c|c|c|c|c|c|}
\hline \multirow{2}{*}{ Chain length (trivial name) } & \multicolumn{9}{|c|}{ g FA/100g fat } \\
\hline & EP2 & E2 & F2 & GSS2 & NN2 & N2 & NE2 & PG2 & SG2 \\
\hline C8:0 (caylic) & 0.34 & 0.50 & 0.17 & 0.16 & 0.39 & 0.45 & 0.45 & 0.16 & 0.56 \\
\hline C10:0 (caproic) & 0.93 & 1.13 & 1.46 & 0.69 & 1.04 & 1.24 & 1.17 & 0.88 & 1.49 \\
\hline C11:0 & 0.00 & 0.00 & 0.17 & 0.00 & 0.00 & 0.00 & 0.00 & 0.00 & 0.00 \\
\hline C12:0 (lauric) & 9.46 & 9.58 & 2.18 & 10.70 & 9.87 & 11.20 & 9.83 & 9.84 & 13.15 \\
\hline $\mathrm{C} 13: 0$ & 0.00 & 0.00 & 0.08 & 0.00 & 0.00 & 0.00 & 0.00 & 0.02 & 0.00 \\
\hline C14:0 (myristic) & 4.57 & 4.59 & 7.67 & 4.60 & 4.75 & 5.39 & 4.64 & 4.83 & 5.56 \\
\hline $\mathrm{C} 15: 0$ & 0.07 & 0.09 & 0.85 & 0.10 & 0.11 & 0.07 & 0.09 & 0.08 & 0.05 \\
\hline C16:0 (palmitic) & 25.22 & 23.40 & 36.77 & 27.02 & 27.45 & 26.83 & 23.80 & 23.31 & 8.26 \\
\hline C18:0 (estearic) & 4.25 & 4.45 & 8.90 & 4.07 & 4.29 & 3.91 & 4.70 & 4.52 & 3.34 \\
\hline C20:0 (arachidic) & 0.35 & 0.34 & 0.29 & 0.37 & 0.39 & 0.33 & 0.33 & 0.41 & 0.27 \\
\hline C21:0 & 0.00 & 0.00 & 0.05 & 0.00 & 0.00 & 0.00 & 0.02 & 0.00 & 0.00 \\
\hline C22:0 (behenic) & 0.18 & 0.17 & 0.09 & 0.13 & 0.14 & 0.18 & 0.23 & 0.16 & 0.52 \\
\hline C23:0 & 0.00 & 0.00 & 0.02 & 0.00 & 0.00 & 0.00 & 0.00 & 0.00 & 0.02 \\
\hline C24:0 (lignoceric) & 0.00 & 0.00 & 0.00 & 0.11 & 0.07 & 0.00 & 0.06 & 0.00 & 0.00 \\
\hline Sum of Saturated & 45.38 & 44.26 & 58.69 & 47.95 & 48.50 & 49.60 & 45.34 & 44.20 & 33.21 \\
\hline C14:1 (myristoleic) & 0.02 & 0.04 & 0.68 & 0.03 & 0.04 & 0.00 & 0.04 & 0.03 & 0.00 \\
\hline C15:1 & 0.00 & 0.00 & 0.00 & 0.00 & 0.00 & 0.00 & 0.00 & 0.00 & 0.00 \\
\hline C16:1 (palmitoleic) & 0.20 & 0.20 & 1.27 & 0.25 & 0.24 & 0.16 & 0.21 & 0.19 & 0.14 \\
\hline C17:1 & 0.05 & 0.06 & 0.21 & 0.06 & 0.06 & 0.04 & 0.06 & 0.07 & 0.06 \\
\hline C18:1n-9 (oleic) & 32.97 & 34.86 & 30.29 & 32.46 & 32.32 & 27.40 & 33.99 & 36.52 & 43.89 \\
\hline C20:1 & 0.17 & 0.17 & 0.11 & 0.24 & 0.23 & 0.20 & 0.16 & 0.39 & 0.21 \\
\hline C22:1n-9 (euricic) & 0.00 & 0.00 & 0.00 & 0.00 & 0.00 & 0.00 & 0.00 & 0.00 & 0.00 \\
\hline C24:1n-9 (nervonic) & 0.00 & 0.00 & 0.00 & 0.00 & 0.00 & 0.00 & 0.00 & 0.04 & 0.00 \\
\hline Sum of MUFAS (cis) & 33.41 & 35.33 & 32.56 & 33.04 & 32.90 & 27.81 & 34.47 & 37.23 & 44.30 \\
\hline C18:2n-6c (linoleic) & 17.64 & 18.18 & 5.73 & 16.51 & 16.44 & 20.38 & 17.97 & 15.90 & 19.99 \\
\hline C18:3n-6 ( $\gamma$-linolenic) & 0.08 & 0.00 & 0.00 & 0.00 & 0.00 & 0.00 & 0.00 & 0.00 & 0.02 \\
\hline $\mathrm{C} 20: 2$ & 0.00 & 0.00 & 0.00 & 0.00 & 0.00 & 0.00 & 0.00 & 0.00 & 0.00 \\
\hline C20:3n-6 (dihomo- $\gamma$-linolenic) & 0.09 & 0.04 & 0.07 & 0.00 & 0.00 & 0.00 & 0.00 & 0.03 & 0.00 \\
\hline C20:4n-6 (Arachidonic) & 0.86 & 0.25 & 0.10 & 0.04 & 0.00 & 0.00 & 0.00 & 0.23 & 0.32 \\
\hline C22:2 & 0.00 & 0.00 & 0.00 & 0.00 & 0.00 & 0.00 & 0.00 & 0.00 & 0.00 \\
\hline Sum of PUFAS n-6 & 18.68 & 18.47 & 5.90 & 16.55 & 16.44 & 20.38 & 17.97 & 16.17 & 20.33 \\
\hline C18:3n3 (linolenic) & 1.87 & 1.52 & 0.35 & 1.73 & 1.56 & 1.97 & 1.76 & 1.92 & 1.67 \\
\hline C20:3n3 & 0.00 & 0.00 & 0.00 & 0.00 & 0.00 & 0.00 & 0.00 & 0.04 & 0.00 \\
\hline C20:5n3 (EPA) & 0.10 & 0.10 & 0.06 & 0.09 & 0.09 & 0.09 & 0.11 & 0.10 & 0.18 \\
\hline C22:6n3 (DHA) & 0.38 & 0.12 & 0.00 & 0.33 & 0.16 & 0.00 & 0.13 & 0.13 & 0.15 \\
\hline Sum of PUFAS n-3 & 2.35 & 1.73 & 0.41 & 2.15 & 1.81 & 2.06 & 2.00 & 2.19 & 2.01 \\
\hline C18:1n-9t (elaidic) & 0.12 & 0.12 & 0.50 & 0.13 & 0.15 & 0.08 & 0.13 & 0.15 & 0.08 \\
\hline C18:2n-6t (linolelaidic) & 0.00 & 0.00 & 0.12 & 0.00 & 0.00 & 0.00 & 0.00 & 0.00 & 0.00 \\
\hline Sum of non-natural TFA & 0.12 & 0.12 & 0.61 & 0.13 & 0.15 & 0.08 & 0.13 & 0.15 & 0.08 \\
\hline C18:1n7t (vaccenic) & 0.06 & 0.10 & 1.37 & 0.12 & 0.14 & 0.07 & 0.09 & 0.07 & 0.08 \\
\hline C18:2n7t (CLA) & 0.00 & 0.00 & 0.46 & 0.06 & 0.07 & 0.00 & 0.00 & 0.00 & 0.00 \\
\hline Sum of natural TFA & 0.06 & 0.10 & 1.83 & 0.18 & 0.21 & 0.07 & 0.09 & $\mathbf{0 . 0 7}$ & 0.08 \\
\hline
\end{tabular}

Data presented as mean. MUFAS: monounsaturated fatty acids, PUFAS: polyunsaturated fatty acids, TFA: trans fatty acids. EP2: Enfapro Premium 2, E2: Equate 2, F2: Friso 2, GSS2: Good Start Supreme 2, NN2: Nan 2, N2: Novamil 2, NE2: Nutra Enfant 2, PG2: Promil, SG2: Similac Gain 2. 
Table 4. Detailed fatty acid profile of stage 3 formulas.

\begin{tabular}{|c|c|c|c|c|c|c|c|c|}
\hline \multirow{2}{*}{ Chain length (trivial name) } & \multicolumn{8}{|c|}{ g FA/100g fat } \\
\hline & EGP3 & E3 & F3 & GP3 & GCS3 & NN3 & NE3 & PG3 \\
\hline C8:0 (caylic) & 0.14 & 0.48 & 0.09 & 0.83 & 0.05 & 0.02 & 0.60 & 0.05 \\
\hline C10:0 (caproic) & 1.42 & 1.07 & 0.46 & 1.63 & 0.14 & 0.11 & 1.28 & 0.12 \\
\hline C11:0 & 0.15 & 0.00 & 0.00 & 0.00 & 0.00 & 0.00 & 0.03 & 0.00 \\
\hline C12:0 (lauric) & 3.18 & 9.20 & 6.51 & 13.50 & 1.01 & 0.68 & 9.22 & 0.65 \\
\hline $\mathrm{C} 13: 0$ & 0.08 & 0.00 & 0.00 & 0.00 & 0.00 & 0.00 & 0.03 & 0.00 \\
\hline C14:0 (myristic) & 7.52 & 4.47 & 3.11 & 5.65 & 1.13 & 1.13 & 4.80 & 0.77 \\
\hline $\mathrm{C} 15: 0$ & 0.81 & 0.13 & 0.07 & 0.05 & 0.10 & 0.10 & 0.16 & 0.08 \\
\hline C16:0 (palmitic) & 22.45 & 9.33 & 25.51 & 8.18 & 25.56 & 27.69 & 10.28 & 16.94 \\
\hline C18:0 (estearic) & 8.00 & 4.02 & 3.56 & 3.21 & 3.96 & 4.11 & 4.19 & 4.36 \\
\hline C20:0 (arachidic) & 0.21 & 0.48 & 0.42 & 0.25 & 0.46 & 0.44 & 0.45 & 0.47 \\
\hline C21:0 & 0.06 & 0.03 & 0.00 & 0.00 & 0.00 & 0.00 & 0.07 & 0.00 \\
\hline C22:0 (behenic) & 0.35 & 0.27 & 0.16 & 0.48 & 0.20 & 0.18 & 0.28 & 0.28 \\
\hline C23:0 & 0.00 & 0.00 & 0.00 & 0.00 & 0.00 & 0.00 & 0.02 & 0.00 \\
\hline C24:0 (lignoceric) & 0.16 & 0.00 & 0.00 & 0.00 & 0.09 & 0.06 & 0.00 & 0.01 \\
\hline Sum of Saturated & 44.54 & 29.46 & 39.90 & 33.78 & 32.69 & 34.50 & 31.40 & 23.74 \\
\hline C14:1 (myristoleic) & 0.68 & 0.08 & 0.00 & 0.00 & 0.02 & 0.03 & 0.11 & 0.01 \\
\hline C15:1 & 0.00 & 0.00 & 0.00 & 0.00 & 0.00 & 0.00 & 0.00 & 0.00 \\
\hline C16:1 (palmitoleic) & 1.07 & 0.28 & 0.24 & 0.13 & 0.27 & 0.25 & 0.34 & 0.22 \\
\hline $\mathrm{C} 17: 1$ & 0.19 & 0.08 & 0.06 & 0.05 & 0.09 & 0.07 & 0.10 & 0.11 \\
\hline C18:1n-9 (oleic) & 39.31 & 39.79 & 42.51 & 43.26 & 42.49 & 39.68 & 38.91 & 40.80 \\
\hline $\mathrm{C} 20: 1$ & 0.14 & 0.71 & 0.44 & 0.21 & 0.44 & 0.33 & 0.68 & 0.55 \\
\hline C22:1n-9 (euricic) & 0.00 & 0.00 & 0.00 & 0.00 & 0.00 & 0.00 & 0.00 & 0.00 \\
\hline C24:1n-9 (nervonic) & 0.00 & 0.08 & 0.00 & 0.00 & 0.00 & 0.00 & 0.08 & 0.02 \\
\hline Sum of MUFAS (cis) & 41.39 & 41.01 & 43.25 & 43.65 & 43.31 & 40.37 & 40.21 & 41.72 \\
\hline C18:2n-6c (linoleic) & 8.84 & 22.56 & 14.24 & 20.37 & 20.85 & 22.18 & 21.41 & 29.80 \\
\hline C18:3n-6 ( $\gamma$-linolenic) & 0.00 & 0.00 & 0.00 & 0.00 & 0.00 & 0.00 & 0.00 & 0.00 \\
\hline C20:2 & 0.00 & 0.06 & 0.00 & 0.00 & 0.00 & 0.00 & 0.06 & 0.05 \\
\hline C20:3n-6 (dihomo- $\gamma$-linolenic) & 0.04 & 0.06 & 0.00 & 0.00 & 0.00 & 0.00 & 0.06 & 0.00 \\
\hline C20:4n-6 (Arachidonic) & 0.06 & 0.14 & 0.14 & 0.05 & 0.05 & 0.00 & 0.14 & 0.10 \\
\hline $\mathrm{C} 22: 2$ & 0.00 & 0.00 & 0.00 & 0.00 & 0.00 & 0.00 & 0.00 & 0.00 \\
\hline Sum of PUFAS n-6 & 8.94 & 22.81 & 14.38 & 20.42 & 20.90 & 22.18 & 21.68 & 29.95 \\
\hline C18:3n3 (linolenic) & 1.43 & 6.13 & 1.98 & 1.71 & 2.41 & 2.42 & 6.04 & 4.15 \\
\hline C20:3n3 & 0.00 & 0.00 & 0.09 & 0.00 & 0.00 & 0.00 & 0.00 & 0.00 \\
\hline C20:5n3 (EPA) & 0.13 & 0.12 & 0.09 & 0.17 & 0.10 & 0.10 & 0.11 & 0.11 \\
\hline C22:6n3 (DHA) & 0.24 & 0.06 & 0.11 & 0.12 & 0.27 & 0.14 & 0.07 & 0.07 \\
\hline Sum of PUFAS n-3 & 1.80 & 6.31 & 2.27 & 2.00 & 2.78 & 2.66 & 6.22 & 4.33 \\
\hline C18:1n-9t (elaidic) & 0.33 & 0.21 & 0.21 & 0.08 & 0.15 & 0.12 & 0.20 & 0.16 \\
\hline C18:2n-6t (linolelaidic) & 0.18 & 0.00 & 0.00 & 0.00 & 0.00 & 0.00 & 0.00 & 0.00 \\
\hline Sum of non-natural TFA & 0.52 & 0.21 & 0.21 & 0.08 & 0.15 & 0.12 & 0.20 & 0.16 \\
\hline C18:1n7t (vaccenic) & 2.05 & 0.13 & 0.00 & 0.07 & 0.10 & 0.12 & 0.20 & 0.09 \\
\hline C18:2n7t (CLA) & 0.77 & 0.06 & 0.00 & 0.00 & 0.06 & 0.06 & 0.09 & 0.00 \\
\hline Sum of natural TFA & 2.82 & 0.20 & 0.00 & 0.07 & 0.16 & 0.18 & 0.29 & 0.09 \\
\hline
\end{tabular}

Data presented as mean. MUFAS: monounsaturated fatty acids, PUFAS: polyunsaturated fatty acids, TFA: trans fatty acids. EGP3: Enfagrow Premium 3, E3: Equate 3, F3: Friso 3, GP3: Gain Plus 3, GCS3: Good Care Supreme 3, NN3: Nan 3 Development, NE: Nutra Enfant 3, PG3: Progress Gold 3. 
Lipid composition requirements proposed by the ESPGHAN are summarized in Table 5. Linoleic and linolenic acids were transformed to grams per $100 \mathrm{kcal}$. Remaining data is reported as $\mathrm{g}$ of fatty acid per $100 \mathrm{~g}$ of total fat. Finally, comparisons of lipid composition between formulas of different stages are shown in Figure 1. Significant differences $(\mathrm{P}<0.05)$ were observed amongst the sum of Lauric and Myristic content, linoleic/linolenic ratio, Omega-3 content, Omega-6/Omevaluga-3 ratio and the MUFA + PUFA/SFA.

Linoleic/Linolenic acid (LA/ALA) ratio in vegetal oil was used as a quality control to determine the coefficient of variation among different methylation and analytical batches. The LA/ALA mean value (standard deviation) of the proposed control was 3.191 (0.052) with a coefficient of variation (CV) of $0.83 \%$. This CV was obtained from 10 measurements corresponding to a 10 day worked period in which all analytical procedures were performed.

\section{Discussion}

The aim of this study was to assess lipid quality and composition of standardized milk formulas marketed in

Table 5. Fat compositional requirements of infant formulas (ESPHAGAN).

\begin{tabular}{|c|c|c|c|c|c|c|c|c|c|}
\hline \multirow{2}{*}{ Formula } & LA & ALA & LA/ALA & $L+M$ & AA & DHA & $a$ TFA & $n$ TFA & $\Sigma$ TFA \\
\hline & \multicolumn{2}{|c|}{ (g/100 kcal) } & \multicolumn{3}{|l|}{ (ratio) } & \multicolumn{2}{|c|}{ (\% of fat) } & & \\
\hline \multicolumn{10}{|c|}{ STAGE1 } \\
\hline Enfamil Premium 1 & 0.85 & 0.082 & 10.4 & 16.5 & 0.70 & 0.30 & 0.13 & 0.07 & 0.19 \\
\hline Equate 1 & 1.00 & 0.091 & 11.0 & 14.0 & 0.19 & 0.16 & 0.09 & 0.06 & 0.15 \\
\hline Frisolac 1 & 0.70 & 0.101 & 6.9 & 9.1 & 0.22 & 0.17 & 0.22 & 0.00 & 0.22 \\
\hline Good Start Supreme 1 & 0.83 & 0.081 & 10.2 & 15.1 & 0.37 & 0.33 & 0.14 & 0.17 & 0.31 \\
\hline Nan 1 & 0.78 & 0.103 & 7.6 & 14.2 & 0.21 & 0.18 & 0.14 & 0.15 & 0.29 \\
\hline Novamil 1 & 1.03 & 0.098 & 10.5 & 19.0 & 0.00 & 0.00 & 0.07 & 0.07 & 0.14 \\
\hline Nutra Enfant 1 & 0.98 & 0.100 & 9.8 & 14.2 & 0.20 & 0.17 & 0.11 & 0.06 & 0.17 \\
\hline Similac 1 & 1.16 & 0.087 & 13.3 & 18.5 & 0.44 & 0.21 & 0.10 & 0.13 & 0.23 \\
\hline SMA Gold 1 & 0.89 & 0.096 & 9.3 & 15.7 & 0.37 & 0.17 & 0.13 & 0.06 & 0.19 \\
\hline \multicolumn{10}{|c|}{ STAGE2 } \\
\hline Enfapro Premium 2 & 0.83 & 0.088 & 9.4 & 14.0 & 0.86 & 0.38 & 0.12 & 0.06 & 0.18 \\
\hline Equate 2 & 0.82 & 0.069 & 11.9 & 14.2 & 0.25 & 0.12 & 0.12 & 0.10 & 0.21 \\
\hline Friso 2 & 0.25 & 0.016 & 15.6 & 9.9 & 0.10 & 0.00 & 0.61 & 1.83 & 2.44 \\
\hline Good Start Supreme 2 & 0.73 & 0.076 & 9.6 & 15.3 & 0.04 & 0.33 & 0.13 & 0.18 & 0.31 \\
\hline NAN2 & 0.72 & 0.068 & 10.6 & 14.6 & 0.00 & 0.16 & 0.15 & 0.21 & 0.35 \\
\hline Novamil 2 & 0.94 & 0.091 & 10.3 & 16.6 & 0.00 & 0.00 & 0.08 & 0.07 & 0.14 \\
\hline Nutra Enfant 2 & 0.81 & 0.080 & 10.1 & 14.5 & 0.00 & 0.13 & 0.13 & 0.09 & 0.22 \\
\hline Promil Gold 2 & 0.67 & 0.080 & 8.4 & 14.7 & 0.23 & 0.13 & 0.15 & 0.07 & 0.22 \\
\hline Similac Gain 2 & 0.99 & 0.083 & 11.9 & 18.7 & 0.32 & 0.15 & 0.08 & 0.08 & 0.16 \\
\hline \multicolumn{10}{|c|}{ STAGE3 } \\
\hline Enfagrow Premium 3 & 0.33 & 0.054 & 6.1 & 10.7 & 0.06 & 0.24 & 0.52 & 2.82 & 3.34 \\
\hline Equate 3 & 0.89 & 0.241 & 3.7 & 13.7 & 0.14 & 0.06 & 0.21 & 0.20 & 0.41 \\
\hline Friso 3 & 0.48 & 0.067 & 7.2 & 9.6 & 0.14 & 0.11 & 0.21 & 0.00 & 0.21 \\
\hline Gain Plus 3 & 1.01 & 0.085 & 11.9 & 19.2 & 0.05 & 0.12 & 0.08 & 0.07 & 0.15 \\
\hline Good Care Supreme 3 & 0.92 & 0.106 & 8.7 & 2.1 & 0.05 & 0.27 & 0.15 & 0.16 & 0.31 \\
\hline NAN 3 & 0.97 & 0.106 & 9.2 & 1.8 & 0.00 & 0.14 & 0.12 & 0.18 & 0.30 \\
\hline Nutra Enfant 3 & 0.84 & 0.237 & 3.5 & 14.0 & 0.14 & 0.07 & 0.20 & 0.29 & 0.49 \\
\hline Prog Gold 3 & 1.00 & 0.139 & 7.2 & 1.4 & 0.10 & 0.07 & 0.16 & 0.09 & 0.25 \\
\hline
\end{tabular}

Data presented as mean. LA: inoleic acid, ALA: inoleniccid, LA/ALA: linoleic acid/linolenic acid ratio, L + M: lauric + myristic acids, AA: arachidonic acid. DHA: docosahexaenoic acid, $a$ TFA: artificial trans fatty acid, nTFA: natural trans fatty acid, TFA: trans fatty acids. 


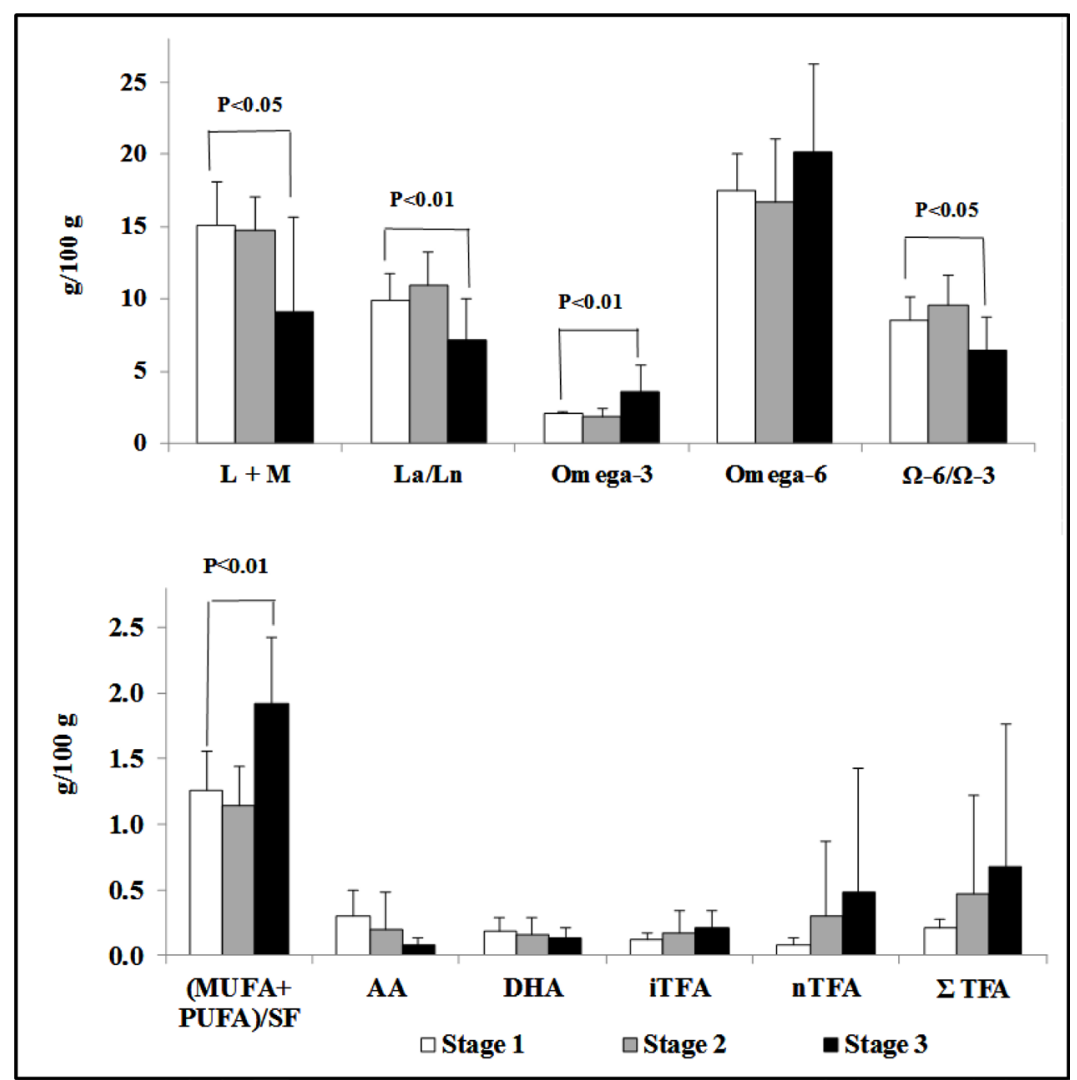

Figure 1. Fat compositional comparisons between formulas from different nutrition stages. L + M: lauric + myristic acids, LA/ALA: linoleic acid/linolenic acid ratio, $\Omega-6 / \Omega-3$ : omega-6/omega-3 ratio, AA: arachidonic acid. DHA: docosahexaenoic acid, $a$ TFA: artificial trans fatty acid, nTFA: natural trans fatty acid, TFA: trans fatty acids.

Mexico according to the ESPHAGAN global recommendations, with an emphasis in TFA. Leading brand products were selected from supermarkets and pharmacies in Mexico City's Metropolitan area during 2011. As shown in Table 5, most of the products tested met with the ESPHAGAN recommendations. Regarding TFA, one product from Stage 3 (Enfagrow Premium 3) exceeded the suggested limit, although the total trans fat load of this product is from natural origin. In view that several authors have reported diverging health effects depending on the source of trans fats, natural or industrial origin, we considered necessary to stratify data in both groups.

The contents of linoleic and linolenic acids were adequate in most formulas; only Friso 2 had lower values of both ( $<0.3 \mathrm{~g} / 100 \mathrm{kcal}$ and $<0.05 \mathrm{~g} / 100 \mathrm{kcal}$, respectively) and Enfagrow Premium 3 showed marginal levels. Two stage 3 formulas (Equate 3 and Nutra Enfant 3) had a linoleic/linolenic acid (LA/ALA) ratio lower than 5:1 and none was higher than 15:1. Likewise, the sum of lauric and myristic acids was lower than $20 \%$ in all formulas; however, two stage 3 formulas showed very low values ( $<2 \%$; Nan 3 and Progress Gold 3). Notably, the content of DHA and AA was insufficient in an important number of products; this is alarming since the intake of omega-3 and omega-6 fatty acids is very low in Mexican population as previously described by Ramirez-Silva et al. [21]. In the succeeding section, formulas are grouped according to their nutrition stage to perform a more exhaustive analysis.

\subsection{Stage 1 Formula}

For infant formulas of stage 1, it was constantly observed a higher proportion of saturated fat $(44.0 \% \pm 5 \%)$ followed by monounsaturated and polyunsaturated fats $(36.3 \% \pm 5.1 \%$ and $19.5 \% \pm 2.5 \%$, respectively). Palmitic acid is the most abundant saturated fatty acid $(22.7 \% \pm 5.7 \%)$. The sum of saturated lauric and myristic acids was similar amongst formulas and was always found within the recommended amount $(<20 \%)$. Oleic acid represents almost the entire content of MUFAs $(36.3 \% \pm 5.1 \%)$. Linoleic and linonelic acids were present in 
adequate amounts. The content of docosahexaenoic acid (DHA) was minor than the allowed value ( $>0.2 \%)$ in $66.6 \%$ of the formulas and arachidonic acid content (AA) was lower than $0.3 \%$ in $55 \%$ of them. AA and DHA were untraceable in Novamil 1. Interestingly, trans fatty acids showed very poor values in all stage 1 formulas $(<0.5 \%)$.

\subsection{Stage 2 Formulas}

The lipid composition of formulas from stage 2 is very similar to those of stage 1 regarding the total content of saturated, monounsaturated and polyunsaturated fats. In like manner, a similar content of the sum of lauric and myristic, linoleic and linolenic acids was observed. The LA/ALA ratio was adequate in all products. Long-chain polyunsaturated fatty acids (LC-PUFA) content remains meager in most products. Low amounts of AA $(<0.3 \%)$ and DHA $(<0.2 \%)$ were found in $78 \%$ of the formulas. Nonetheless, it is important to consider that the formulas of stage 2 are recommended for children who have already begun the process of weaning and might consume LC-PUFA from other food sources. All formulas had very low values of total trans fatty acids $(<0.5 \%)$, except Friso 2 (2.44\%).

\subsection{Stage 3 Formulas}

Formulas from sage 3 have a similar fatty acid profile compared to the formulas of stages 1 and 2. Contrastingly, stage 3 formulas showed a higher variation in the sum of lauric and myristic acids; three products reported a scant proportion $(<2 \%)$ of these fatty acids. Regarding TFA content, just one formula (Enfagrow Premium 3 ) displayed a value greater than $3 \%$; the rest had values lower than $0.5 \%$. In relation to AA and DHA content, almost all the formulas present consistently low levels.

\section{Conclusion}

Most of the analyzed formulas that are marketed in Mexico comply with the ESPHAGAN global recommendations. TFA were detected in remarkably small quantities and below the suggested limit $(<3 \%)$ in almost all formulas. Regarding LC-PUFA, it was intriguing to find that the content of DHA and AA was generally inadequate.

\section{Disclosure Statement}

The authors declare no potential conflicts of interest.

\section{Financial Support}

This study was funded by the Office of Graduate Studies and Research at the University La Salle, Mexico City. Registration number: Q082-09.

\section{References}

[1] American Academy of Pediatrics (2012) Breastfeeding and the Use of Human Milk. Pediatrics, 19, 827-841.

[2] Heird, W.C. (2007) Progress in Promoting Breast-Feeding, Combating Malnutrition, and Composition and Use of Infant Formula, 1981-2006. The Journal of Nutrition, 137, 499-502.

[3] González de Cosío, T., Escobar-Zaragoza, L., González-Castell, L.D. and Rivera-Dommarco, J.A. (2013) Infant Feeding Practices and Deterioration of Breastfeeding in Mexico. Public Health of Mexico, 55, 170-179.

[4] Koletzko, B., Baker, S., Cleghorn, G., Neto, U.F., Gopalan, S., Hernell, O., Hock, Q.S., Jirapinyo, P., Lonnerdal, B., Pencharz, P., Pzyrembel, H., Ramirez-Mayans, J., Shami, R., Turck, D., Yamashiro, Y. and Ding, Z.-Y. (2005) Global Standard for the Composition of Infant Formula: Recommendations of an ESPGHAN Coordinated International Expert Group. Journal of Pediatric Gastroenterology \& Nutrition, 41, 584-599. http://dx.doi.org/10.1097/01.mpg.0000187817.38836.42

[5] Auestad, N., Scott, D.T., Janowsky, J.S., Jacobsen, C., Carroll, R.E., Montalto, M.B. and Connor, S.L. (2003) Visual, Cognitive, and Language Assessments at 39 Months: A Follow-Up Study of Children Fed Formulas Containing LongChain Polyunsaturated Fatty Acids to 1 Year of Age. Pediatrics, 112, 177-183. http://dx.doi.org/10.1542/peds.112.3.e177

[6] Uauy, R., Hoffman, D.R., Mena, P., Llanos, A. and Birch, E.E. (2003) Term Infant Studies of DHA and ARA Supple- 
mentation on Neurodevelopment: Results of Randomized Controlled Trials. The Journal of Pediatrics, 143, 17-25. http://dx.doi.org/10.1067/S0022-3476(03)00398-6

[7] Birch, E.E., Garfield, S., Hoffman, D.R., Uauy, R. and Birch, D.G. (2000) A Randomized Controlled Trial of Early Dietary Supply of Long-Chain Polyunsaturated Fatty Acids and Mental Development in Term Infants. Developmental Medicine \& Child Neurology, 42, 174-181. http://dx.doi.org/10.1111/j.1469-8749.2000.tb00066.x

[8] Koletzo, B., Agostoni, C., Carlson, S.E., Clandinin, T., Hornstra, G., Neuringer, M., Uauy, R., Yamashiro, Y. and Willatts, P. (2001) Long Chain Polyunsaturated Fatty Acids (LC-PUFA) and Perinatal Development. Acta Paediatrica, 90, 460-464. http://dx.doi.org/10.1111/j.1651-2227.2001.tb00452.x

[9] Hunter, J.E. (2005) Dietary Levels of Trans-Fatty Acids: Basis for Health Concerns and Industry Efforts to Limit Use. Nutrition Research, 25, 499-513. http://dx.doi.org/10.1016/j.nutres.2005.04.002

[10] Fritsche, J. and Steinhart, H. (1998) Analysis, Occurrence, and Physiological Properties of Trans Fatty Acids (TFA) with Particular Emphasis on Conjugated Linoleic Acid Isomers (CLA)—A Review. European Journal of Lipid Science and Technology, 100, 190-210. http://dx.doi.org/10.1002/(SICI)1521-4133(199806)100:6<190::AID-LIPI190>3.0.CO;2-5

[11] Lichtenstein, A.H. (1998) Trans Fatty Acids and Blood Lipid Levels, Lp (A), Parameters of Cholesterol Metabolism and Hemostatic Factors. The Journal of Nutritional Biochemistry, 9, 244-248. http://dx.doi.org/10.1016/S0955-2863(98)00016-3

[12] Salo, P., Seppänen-Laakso, T., Laakso, I., Seppänen, R., Niinikoski, H., Viikari, J. and Simell, O. (2000) Low Saturated Fat, Low-Cholesterol Diet in 5-Year-Old Children: Effect on Intake and Composition of Trans Fatty Acids and Other Fatty Acids in Serum Phospholipid Fraction-The STRIP Study. The Journal of Pediatrics, 136, 46-52. http://dx.doi.org/10.1016/S0022-3476(00)90048-9

[13] Martínez, R.G., Martínez, B.A., Salas, F.A. and Maldonado, H.J. (2013) Association between Metabolic Syndrome and Erythrocyte Fatty Acid Profile in Mexican Adolescents: A Trans Fatty Acid Approach. Food and Nutrition Sciences, 4, 51-58. http://dx.doi.org/10.4236/fns.2013.49A1009

[14] Lauer, J.A., Betrán, A.P., Barros, A.J. and de Onís, M. (2006) Deaths and Years of Life Lost Due to Suboptimal Breast-Feeding among Children in the Developing World: A Global Ecological Risk Assessment. Public Health Nutrition, 9, 673-685. http://dx.doi.org/10.1079/PHN2005891

[15] Lauer, J., Beltrán, A., Victora, C., de Onis, M. and Barros, A. (2004) Breastfeeding Patterns and Exposure to Suboptimal Breastfeeding among Children in Developing Countries: Review and Analysis of Nationally Representative Surveys. BMC Medicine, 2, 26. http://dx.doi.org/10.1186/1741-7015-2-26

[16] Barrio, J., Díaz-Martín, J., Manrique, I., Martín, M.B. and Ortega, E. (2015) Expert Consensus on the Nutritional Aspects of Initial and Follow-On Infant Formulas. Anales de Pediatría, 83, 376-386. http://dx.doi.org/10.1016/j.anpede.2015.09.022

[17] Hamilton, R.J. and Hamilton, S. (1994) Lipid Analysis: A Practical Approach. Oxford University Press, New York. http://dx.doi.org/10.1046/j.1471-4159.1995.t01-3-64031424.x

[18] Kramer, J., Fellner, V., Dugan, M., Sauer, F., Mossoba, M. and Yurawecz, M. (1997) Evaluating Acid and Base Catalysts in the Methylation of Milk and Rumen Fatty Acids with Special Emphasis on Conjugated Dienes and Total Trans Fatty Acids. Lipids, 32, 1219-1228. http://dx.doi.org/10.1007/s11745-997-0156-3

[19] AOAC (1998) Fatty Acids in Oils and Fats.Preparation of Methyl Esters. Boron Trifluoride Method. Official Method 969.33. AOAC International, Rockville.

[20] AOCS (1997) Preparation of Methyl Esters of Long-Chain Fatty Acids. Official Method Ce 2-66. AOCS, Urbana.

[21] Ramírez, S.I., Villalpando, S., Moreno, S.J. and Bernal, M.D. (2011) Fatty Acids Intake in the Mexican Population. Results of the National Nutrition Survey 2006. Nutrition and Metabolism, 8, 1-10.

http://dx.doi.org/10.1186/1743-7075-8-33 